\title{
Skin permeability barrier and occlusion: no delay of repair in irritated human skin*
}

\author{
J. WeLzel, K. P. WiLheLM ${ }^{1}$ AND H. H. WolfF \\ Department of Dermatology, Medical University of Lübeck, Germany \\ ${ }^{1}$ proDERM Institute for Applied Dermatological Research, Hamburg, Germany
}

\begin{abstract}
It has been reported that occlusive treatment of irritated skin results in a reduction of barrier repair activities in hairless mice. In contrast, the clinically observed benefit of occlusion in the treatment of hand eczema and other chronic skin diseases with a perturbed barrier function is well-known. While the beneficial effect of occlusion has been proven for the treatment of psoriasis there are no controlled clinical studies of the effect of occlusion on irritated human skin. We have therefore evaluated the effect of various occlusive treatments on repair of the human skin permeability barrier under controlled experimental conditions. Barrier perturbation was induced either by application of sodium lauryl sulfate (SLS) or by repeated tape stripping. This was followed by treatment with different occlusive and semiper meable dressings, partly after pre-treatment with petrolatum. Repair of water barrier function was evaluated by daily measurements of transepidermal water loss (TEWL) for 1 week. SLS irritation and tape stripping led to a 6-fold increase in TEWL as a sign of severe water barrier perturbation, followed by a stepwise decrease over the following days. Occlusion did not significantly delay barrier repair as measured by TEWL. Only in tape-stripped skin did TEWL stay at high levels during treatment with self-adhesive dressings. This may be explained by damage of newly formed stratum corneum caused by changing of these membranes. Our results indicate that, in contrast to earlier observations in hairless mouse skin, permeability barrier repair activities are not significantly delayed by occlusive treatment in human skin.
\end{abstract}

Occlusive treatment has positive effects in a variety of skin diseases. It is a well-established therapy in wound healing and in chronic inflammatory skin diseases with perturbed barrier function (1-3). On the other hand, experimental investigations in hairless mice have recently shown that artificial restoration of the water barrier by occlusion resulted in a delay of epidermal repair activities. The stimulation of DNA and lipid synthesis for regeneration of the permeability barrier after disturbance was reduced under occlusive conditions and delayed dependent on the degree of occlusion (4-6). These effects were demonstrated in hairless mice and were in contrast to the clinical experience of accelerated healing using occlusive dressings.

Animal models are of minor advantage for

* Presented in part at the 2nd International Symposium on Irritant Contact Dermatitis, 14-16 April, 1994, Zürich, Switzerland. studying the barrier function of the skin, because there are structural and functional differences from human skin (7-8). It was therefore our objective to evaluate the influence of occlusion on skin permeability barrier repair in human skin under controlled experimental conditions.

2 different kinds of barrier abrogation, either by irritation with the anionic surfactant sodium lauryl sulfate (SLS) or by removal of the stratum corneum by means of repeated tape stripping, were investigated. For comparison with the studies in hairless mice, in which a short time and single barrier disturbance was investigated, we chose an acute irritation model. For occlusion we used several membranes with different water vapor permeability, to look for effects that might be dependent on the degree of occlusion. To simulate clinical conditions, the 1st experiment was repeated with a combination of topical therapy and occlusion. Therefore, the irritated areas were first 
treated with petrolatum as a standard vehicle before covering them with the membranes.

\section{Occlusive dressings}

\section{Materials and Methods}

4 membranes with different characteristics were used:

OpSite ${ }^{\circledR}$

semipermeable, self-adhesive

(Smith \& Nephew Medical Ltd., Hull, UK)

Tegaderm $^{\circledR}$ : $\quad$ semipermeable, self-adhesive (3M, St.Paul, Minn., USA)

Gore-Tex ${ }^{\circledR}$ semipermeable, non-adhesive (W.L.Gore \& Associates GmbH, Putzbrunn, Germany)

Polyethylene nonpermeable, non-adhesive

foil: (Melitta $\mathrm{GmbH}$, Minden-Dützen, Germany)

The water vapor permeability of the occlusive and semipermeable dressings was evaluated by measuring the water evaporation from an evaporation standard (9) (petri dish filled with $10 \mathrm{ml}$ water). The membranes were placed on the petri dishes without extension. The measurements were repeated on several days.

Gore-Tex had the highest water vapor permeability, with TEWL values of about $26 \mathrm{~g} / \mathrm{m}^{2} \mathrm{~h}$. OpSite and Tegaderm were less vapor permeable, with values of about 10 and $7 \mathrm{~g} / \mathrm{m}^{2} \mathrm{~h}$, respectively. Polyethylene (PE) foil showed the lowest values (about $2 \mathrm{~g} / \mathrm{m}^{2} \mathrm{~h}$ ) and was nearly impermeable.

The occlusive effect of petrolatum was demonstrated by TEWL measurements of healthy skin before, $10 \mathrm{~min}$, and $1 \mathrm{~h}$ after treatment. Petrolatum decreased the TEWL immediately after application to about $20 \%$ of baseline values in healthy skin. After $1 \mathrm{~h}$, the TEWL returned to nearly normal values.

Sodium lauryl sulfate was of analytical grade purity (>99\%, E. Merck, Darmstadt, Germany).

For tape stripping we used Tesa ${ }^{\circledR}$ (No. 4104, Beiersdorf, Hamburg, Germany).

\section{Experiment no. 1: Skin irritation and occlusion}

10 healthy female volunteers (age 22-43 years, nonatopics) were treated with sodium lauryl sulfate (SLS) to disturb stratum corneum barrier function (day 1). $200 \mu \mathrm{l}$ of $20 \mathrm{mM}(\cong 0.5 \%)$ aqueous solution were applied to 6 areas on the volar side of the forearm. Occlusive polypropylene chambers (diameter $25 \mathrm{~mm}$, Hilltop, Cincinnati, Ohio, USA) with the irritant were fixed with nonocclusive tape (Scanpor, Norgesplaster, Vennesla, Norway) and remained on the skin for $24 \mathrm{~h}$. The skin was then carefully washed with water and dried with a soft paper towel to remove the remains of irritant.

$1 \mathrm{~h}$ after removal of the irritant, the areas were covered with the different occlusive and semipermeable membranes for $2 \times 23 \mathrm{~h}$ (days 2 and 3). One area was left unoccluded as control. Treatments rotated on the test sites between volunteers to avoid an anatomical selection bias.

Measurements of TEWL were done on day 1 before treatment, on day $21 \mathrm{~h}$ after removal of the irritant, and on days 3 and $4 \mathrm{l} \mathrm{h}$ after removal of the occlusive dressings. The last measurement was done after $24 \mathrm{~h}$ without any treatment on day 5 . In this experiment, daily visual scores of erythema and dryness were determined additionally.

\section{Experiment no. 2: skin irritation and petrolatum/oc- clusion}

10 healthy volunteers ( 7 female, 3 male, age $22-47$ years, nonatopics) were pretreated on 4 areas on the volar side of the forearm with SLS, as described above. On day 2, the irritant was washed off. After 1 h, 3 areas were treated with pure white petrolatum $\left(7 \mathrm{mg} / \mathrm{cm}^{2}\right)$ : one test site was occluded with Gore-Tex, another with PE foil, and the 3rd left unoccluded. The 4th irritated area remained untreated as control. The treatments rotated between the subjects. Measurements of TEWL were performed daily: before irritation, $1 \mathrm{~h}$ after irritation, $1 \mathrm{~h}$ after treatment, on day 5 without any treatment.

\section{Experiment no. 3: tape stripping and occlusion}

In 10 healthy volunteers ( 9 female, 1 male, age 2240 years, nonatopics) on 5 areas of the volar aspect of the forearm, the stratum corneum was partially removed by repeated (30-40X) tape stripping, controlled by an increase of TEWL up to at least 30 $\mathrm{g} / \mathrm{m}^{2}$ h. 4 areas were covered with the occlusive membranes for $2 \times 23 \mathrm{~h}$, one area being left unoccluded as control. Treatment rotated between volunteers.

TEWL was measured on day 1 before and after tape stripping, on day 2 and $31 \mathrm{~h}$ after removal of the dressings and on day 4 and 5 without any treatment.

We used the Tewameter (TM 210, Courage \& Khazaka, Cologne, Germany) for TEWL measurements. The volunteers rested for $20 \mathrm{~min}$ in a room with an ambient air temperature of $20 \pm 1^{\circ} \mathrm{C}$ and a relative air humidity of $50 \pm 5 \%$, before performing the measurements. The study design was approved by the ethical committee of the Medical University of Lübeck.

Statistical analysis was done using the Friedman 
rank variance analysis with consecutive Wilcoxon/ Wilcox comparisons (10). The values of the different treatments were compared with the untreated control.

\section{Results}

\section{Experiment no. 1}

Treatment with SLS induced a defect of the permeability barrier as shown by a 6-fold increase of TEWL (Fig. 1). The barrier repair process could be observed over the following days by a stepwise decrease of TEWL values. On the last day of investigation, TEWL still remained slightly elevated compared to pre-irritation values. The decrease of

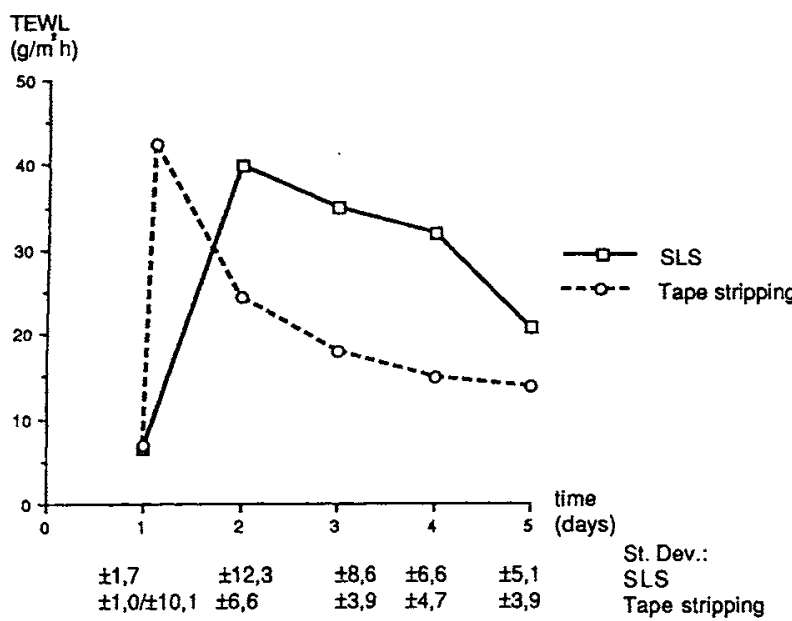

Fig. 1. TEWL on the control site after irritation with SLS and after tape stripping. The degree of barrier perturbation, as measured by TEWL, was comparable independent of the different mode of disturbance.

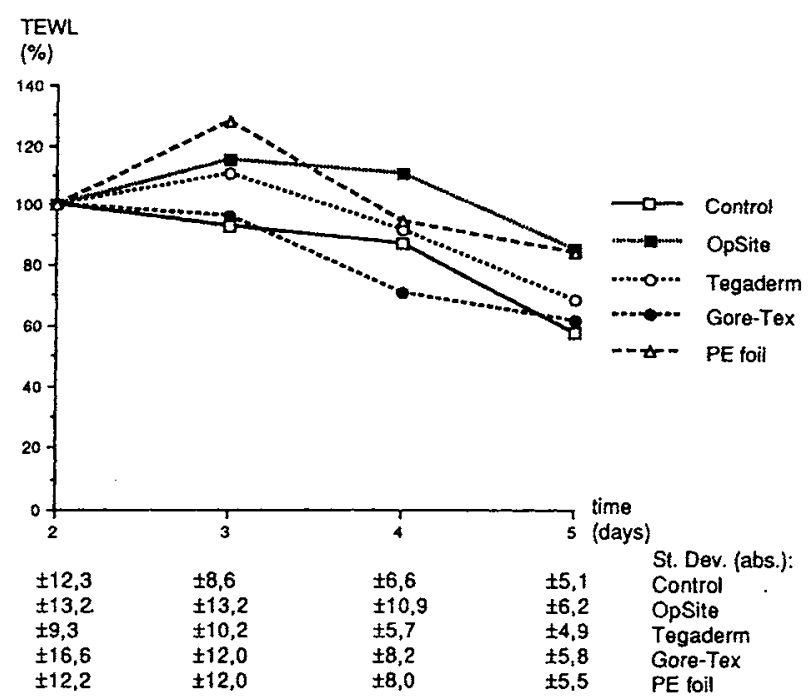

Fig. 2. TEWL after irritation with SLS on day 1, occlusive treatment on days 2 and 3 . There were no significant differences between the control site and the occluded areas.

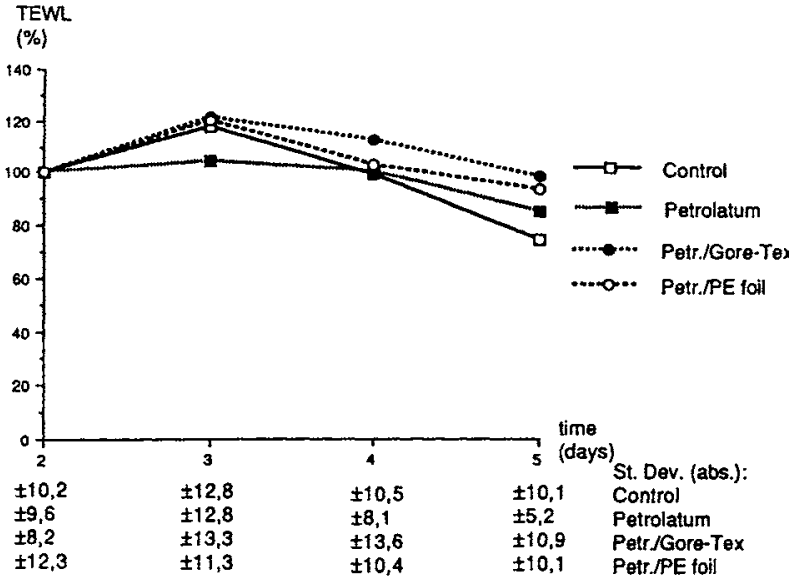

Fig. 3. TEWL after irritation with SLS on day 1, treatment with petrolatum, partly with occlusive dressings on days 2 and 3. There were no significant differences between the control site and the occluded areas.

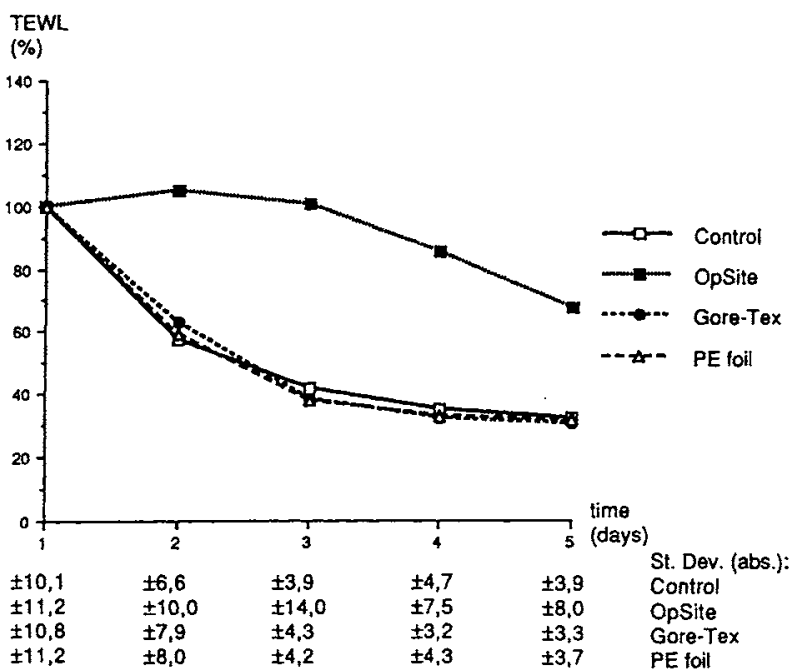

Fig. 4. TEWL after tape stripping on day 1 , occlusive treatment on days 1 and 2. Treatment with OpSite led to a delay of TEWL decrease.

TEWL was not influenced by the different postirritation treatments with the occlusive dressings. . There was no significant difference between the various membranes and the unoccluded control site. Furthermore, the different water vapor permeability of the membranes did not have any influence upon TEWL decrease (Fig. 2).

The SLS-induced visible skin irritation could be demonstrated by an immediate erythema reaction on day 2 and a delayed increase of dryness scores with a maximum on day 4 . Occlusive conditions slightly diminished the visible changes under most of the dressings except PE foil, which did not improve the scaling. The difference between OpSite and the control was significant for dryness and erythema on day 4 . 


\section{Experiment no. 2}

The increase of TEWL after SLS irritation lasted for up to day 3 , irrespective of the different treatments, before decreasing over the following days. On day 4 , after 2 days of treatment, TEWL values again reached the same level as on day 2 directly after irritation. This course could be observed in all areas. There was no significant difference between the control area, the petrolatum-treated test site and the additionally occluded areas. Furthermore, the degree of occlusion of the different membranes, low for Gore-Tex and high for PE foil, had no influence upon TEWL (Fig. 3).

\section{Experiment no. 3}

Directly after tape stripping, TEWL increased from about $7 \mathrm{~g} / \mathrm{m}^{2} \mathrm{~h}$ up to $40 \mathrm{~g} / \mathrm{m}^{2} \mathrm{~h}$ because of the partial removal of the stratum corneum (Fig. 1). The regeneration of the barrier could be observed by a rapid decrease of TEWL over the following days. Even at the control site, however, until day 5, TEWL did not reach baseline values and stayed slightly elevated. Some of the membranes had no influence upon TEWL decrease, whereas the treatment with OpSite led to a delay of TEWL normalization. OpSite was a self-adhesive membrane, in contrast to the other occlusive dressings which were used in this experiment. The difference between TEWL values of OpSite-treated areas and the unoccluded control was statistically significant at day $4(p<0,05)$. TEWL started to decrease only after ending the occlusive treatment with this membrane. Treatment with Gore-Tex and PE foil did not influence the TEWL decrease, despite their different water vapor permeability (Fig. 4).

\section{Discussion}

Skin contact with sodium lauryl sulfate leads to a barrier perturbation with keratin denaturation and fluidization of intercellular lipids. The permeability barrier of the skin is disturbed without any loss of stratum corneum $(11,12)$. In higher concentrations and with longer application time, an inflammatory reaction with spongiosis and exocytosis starts, followed by parakeratosis and acanthosis.

It is well-known that skin irritation with SLS can be demonstrated by an increase of TEWL (14, 15). Our experiments showed that TEWL normalization as a parameter for water barrier integrity and repair was not influenced by occlusive treatment. Application of various occlusive and semipermeable dressings led to no further irritation or delay of barrier repair, irrespective of the different water vapor permeability of the membranes. Furthermore, petrolatum plus occlusion did not influence repair time after acute skin irritation.

Tape stripping removes layers of the stratum corneum and causes a barrier defect. It leads to hyperproliferation without severe inflammation (16-18).

In untreated skin, TEWL increased after tape stripping for some days because of the loss of parts of the stratum coneum. The high values were caused by the remaining fully hydrated basal layers of the stratum corneum, and returned to normal values during regeneration of the barrier. Occlusion as an artificial restoration of the permeability barrier showed different results in tapestripped skin: Gore-Tex and PE foil had no influence upon TEWL, whereas treatment with OpSite delayed TEWL decrease. This effect could not be explained by the water vapor permeability of the membranes, which was high for Gore-Tex, medium for OpSite and low for PE foil. The delay of barrier repair seemed to depend on another difference between these foils. OpSite was self-adhesive, and the daily change of this membrane might have removed newly formed layers of the stratum corneum. It is likely that this additional tape-strippinglike artificial effect caused a further perturbation of the barrier and was responsible for the delay of TEWL decrease. The newly formed thin stratum corneum after tape stripping seemed to be less resistant against adhesives than the SLS-irritated skin with a normal or increased horny layer.

In summary, our results indicate that occlusion does not delay barrier repair in irritated human skin. Occlusive treatment had no marked influence upon healing after acute short-time irritation. It neither additionally irritated the skin nor accelerated the repair process.

This clearly contrasts with some previously reported data. Investigations in hairless mice showed a further deterioration of the barrier function by occlusion in both the tape-stripping and the SLSirritation model. The integrity of the epidermal permeability barrier is known to have an influence upon DNA and lipid synthesis and lamellar body secretion (4-6). Defects of the barrier with increased transepidermal water loss are accompanied by a burst of synthesis $(19,20)$. This was interpreted as barrier repair activity. Occlusive treatment after barrier disruption as an artificial restoration of the barrier, delayed DNA and lipid synthesis, dependent on the water vapor permeability of the dressings. Some other investigations in human skin demonstrated an increase of TEWL after occlusion of healthy skin, and a further barrier perturbation of SLS-irritated skin under occlusive conditions $(21,22)$. 
Investigations on the effect of occlusion on skin permeability barrier should consider some side-effects of the treatment. Occlusive treatment increases the hydration of the stratum corneum (23, 24). We found high values for electrical capacitance for longer than $1 \mathrm{~h}$ after removal of the membranes. This hyperhydration should be considered when determining the TEWL. The elevated TEWL, which was found for hours after occlusive therapy (25) and interpreted as irritation, might only be due to prolonged evaporation of excess water bound in the stratum corneum. This relationship, with a positive correlation between water evaporation and the hydration state of the skin, was confirmed by investigations of Tagami (26).

The irritant should be washed off after application, before starting the occlusive treatment, to avoid further influence of the irritant on the barrier function or enhanced penetration into the skin. Van der Valk \& Maibach (22) found an increased irritant response to SLS under occlusion. In this study, the membranes were applied onto the skin directly after the irritation without washing off the SLS. Under these conditions, it is more than likely that some remaining irritant under occlusion caused the observed effects. Moreover, in this study and in the investigations in hairless mice, the TEWL was measured $10 \mathrm{~min}$ after removing the membranes, when water release from the hyperhydrated stratum corneum influenced the values rather than a real water barrier defect.

In our study, we were able to demonstrate that occlusion of various degrees did not have a deleterious effect upon barrier repair activity in human skin after acute and short-time irritation. However, neither did occlusion have a positive effect upon barrier repair time, as was expected from clinical experience in chronic skin diseases.

Occlusion is known to have an antiproliferative and anti-inflammatory effect upon hyperproliferative skin diseases $(27,28)$. Chronic and repeated barrier damage, as in hand eczema, leads to an excessive and pathologic hyperproliferation, which may itself result in high transepidermal water loss. Occlusion seems to modulate the barrier repair activities without stopping them totally. Acute shorttime irritation is followed only by a mild proliferation, which occlusion does not greatly influence (29).

Particularly in psoriasis, the benefit of occlusion has been well demonstrated $(30,31)$. Most of these studies used self-adhesive membranes, and it has been proposed that the improvement of the induration and the scaling might be a mechanical effect of stripping away the scales (1). This was supported by the observation that the time of appli- cation, short-time versus prolonged occlusion, was not very important to the final result (3).

In earlier studies, hairless mice were used for investigation of irritant contact dermatitis and occlusive effects (4-6). When interpreting these results, some differences between the animal model and human skin should be considered. The stratum corneum of the hairless mouse skin was found to be more fragile and less resistant to hydration than human skin. Prolonged occlusion itself damaged the stratum corneum barrier of the hairless mouse but not of human skin $(7,8)$. It is more than likely that a pre-damaged stratum corneum, either by surfactant exposure or by tape stripping, will be even more susceptable to this hydration damage.

Our results suggest that the hairless mouse may be a limited model for the study of barrier function of human skin, especially in combination with hyperhydration and occlusion.

\section{Acknowledgement}

Supported by a grant from the Deutsche Forschungsgemeinschaft (DFG Wi 879/3-1).

\section{References}

1. Van Vlijmen-Willems I M J J, Chang A, Boezeman J B M, van de Kerkhof P C M. The immunhistochemical effect of a hydrocolloid occlusive dressing (DuoDERM E) in psoriasis vulgaris. Dermatology 1993: 187: 257-262.

2. Mennen U, Wiese A. Fingertip injuries management with semiocclusive dressing. $J$ Hand Surg 1993: 18: 416-422

3. Wollina U, Knopf B, Fünfstück V et al. Okklusivtherapie der Psoriasis - Vergleich der klinischen Wirksamkeit von kurzzeitiger und prolongierter Anwendung. $Z$ Hautkr 1990: 65: 737-739.

4. Proksch E, Feingold K R, Mao-Qiang M, Elias P M. Barrier function regulates epidermal DNA synthesis. $J$ Clin Invest 1991: 87: 1668-1673.

5. Proksch E. Regulation der epidermalen Permeabilitätsbarriere durch Lipide und durch Hyperproliferation. Hautarzt 1992: 43: 331-338.

6. Proksch E, Feingold K R, Elias P M. Epidermal HMG CoA reductase activity in essentially fatty acid deficiency: Barrier requirements rather than eicosanoid generation regulate cholesterol synthesis. $J$ Invest Dermatol 1992: 99: 216-220.

7. Bond J R, Barry B W. Limitations of hairless mouse skin as a model for in vitro permeation studies through human skin: Hydration damage. J Invest Dermatol 1988: 90: 486489.

8. Bond J R, Barry B W. Hairless mouse skin is limited as a model for assessing the effects of penetration enhancers in human skin. J Invest Dermatol 1988: 90: 810-813.

9. Pinnagoda J, Tupker R A, Agner T, Serup J. Guidelines for transepidermal water loss (TEWL) measurement. Contact Dermatitis 1990: 22: 164-178.

10. Sachs L. Angewandte Statistik, 6th edition. Berlin: Springer, 1991: 673-675.

11. Willis C M, Stephens C J M, Wilkinson J D. Preliminary findings on the patterns of epidermal damage induced by 
irritants in man. In: Frosch P J, Dooms-Goossens A, Lachapelle J M, Rycroft R J G, Scheper R J (eds): Current topics in Contact Dermatitis. Berlin, Heidelberg, New York, London, Paris, Tokyo, Hong Kong: Springer, 1989; 42-45.

12. Wilhelm K P, Cua A, Wolff $H H$, Maibach $H$ I. Surfactant induced stratum corneum hydration in vivo: prediction of the irritation potential of anionic surfactants. $J$ Invest Dermatol 1993: 101: 310-315.

13. Wilhelm K P, Freitag G, Wolff $H H$. Surfactant-induced skin irritation and skin repair: Evaluation of the acute human irritation model by noninvasive techniques. $\mathrm{J} \mathrm{Am}$ Acad Dermatol 1994: 30: 944-949.

14. Wilhelm K P, Freitag G, Wolff $H$ H. Surfactant-induced skin irritation and skin repair: Evaluation of a cumulative human irritation model by noninvasive techniques. $J \mathbf{A m}$ Acad Dermatol 1994: 31: 981-987.

15. Lee $\mathrm{C} \mathrm{H}$, Maibach $\mathrm{H}$ I. The sodium lauryl sulfate model: an overview. Contact Dermatitis 1995: 33: 1-7.

16. Hashimoto $Y$, Tsutsui $M$, Iizuka $H$. Flow cytometric analysis of pig epidermal keratinocytes: Effect of tape stripping. J Dermatol Sci 1992: 4: 193-201.

17. Rijzewijk J J, Boezeman J B M, Bauer F W. Synchronized growth in human epidermis following tape stripping: its implication for cell kinetic studies. Cell Tissue Kinet 1988: 21: 227-229.

18. Gerritsen M J P, Van Erp P E J, Van Vlijmen-Willems I M $\mathrm{J} J$ etal. Repeated tape stripping of normal skin: a histological assessment and comparison with events seen in psoriasis. Arch Dermatol Res 1994: 286: 455-461.

19. Menon G K, Feingold K R, Elias P M. Lamellar body secretory response to barrier disruption. $J$ Invest Dermatol 1992: 98: 279-289.

20. Holleran W M, Feingold K R, Mao-Qiang M et al. Regulation of epidermal sphingolipid synthesis by permeability barrier function. $J$ Lipid Res 1991: 32: 1151-1158.

21. Kligman A M. Hydration injury to human skin. In: Elsner P, Berardesca E, Maibach H I (eds): Bioengineering and the skin: water and the stratum corneum. Boca Raton, Ann Arbor, London, Tokyo: CRC Press, 1994; 251- 255.

22. Van der Valk P G M, Maibach H I. Post-application occlusion substantially increases the irritant response to the skin to repeated short-term sodium lauryl sulfate (SLS) exposure. Contact Dermatitis 1989: $21: 335-338$.

23. Anderson $R$ L, Cassidy J M, Hansen J R, Yellin W. The effect of in vivo occlusion on human stratum corneum hy- dration-dehydration in vitro. $J$ Invest Dermatol 1973: 61: 375-379.

24. Mikulowska A. Reactive changes in human epidermis following simple occlusion with water. Contact Dermatitis 1992: 26: 224-227.

25. Agner T, Serup J. Time course of occlusive effects on skin evaluated by measurements of transepidermal water loss (TEWL). Contact Dermatitis 1993: 28: 6-9.

26. Tagami $H$. Impedance measurement for evaluation of the hydration state of the skin surface. In: Leveque J L (ed): Cutaneous investigation in health and disease. New York: Marcel Dekker Inc., 1990: 79-111.

27. Fisher L B, Maibach H I. The effect of occlusive and semipermeable dressings on the mitotic activity of normal and wounded human epidermis. Br J Dermatol 1972: 86: 593600.

28. Gottlieb A B, Staiano-Coico L, Cohen S R et al. Occlusive hydrocolloid dressings decrease keratinocyte population growth fraction and clinical scale and skin thickness in active psoriatic plaques. J Dermatol Sci 1990: 1: 93-96.

29. Wood L C, Elias P M, Sequeira-Martin S M et al. Occlusion lowers cytokine mRNA levels in essential fatty acid-deficient and normal mouse epidermis, but not after acute barrier disruption. I Invest Dermatol 1994: 103: 834 838.

30. Kragballe K, Larsen F G. A hydrocolloid occlusive dressing plus triamcinolone acetonide cream is superior to clobetasol cream in palmo-plantar pustulosis. Acta Dermatovenereologica 1991: 71: 540-542.

31. Broby-Johansen U, Karlsmark T, Petersen L J, Serup J. Ranking of the antipsoriatic effect of various topical corticosteroids applied under a hydrocolloid dressing - skinthickness, blood-flow and colour measurements compared to clinical assessments. Clin Exp Dermatol 1990: 15: 343348.

Address:

Julia Welzel

Klinik für Dermatologie und Venerologie

Medizinische Universität zu Lübeck

Ratzeburger Allee 160

23538 Lübeck

Germany 